\title{
Blockade of S100A3 activity inhibits murine hair growth
}

W. Guan', Q. Deng', X.L. Yu', Y.S. Yuan', J. Gao², J.J. Li' ${ }^{2}$, L. Zhou', P. Xia', G.Y.Q. $\operatorname{Han}^{3}$, W. Han' ${ }^{2}$ and Y. Yu'

${ }^{1}$ Shanghai Municipality Key Laboratory of Veterinary Biotechnology, School of Agriculture and Biology, Shanghai Jiao Tong University, Shanghai, China ${ }^{2}$ Laboratory of Regeneromics, School of Pharmacy, Shanghai Jiao Tong University, Shanghai, China

${ }^{3}$ University High School, Irvine, CA, USA

Corresponding authors: Y. Yu / W. Han

E-mail: yanyu@sjtu.edu.cn / weihan@sjtu.edu.cn

Genet. Mol. Res. 14 (4): 13532-13544 (2015)

Received March 10, 2015

Accepted July 23, 2015

Published October 28, 2015

DOI http://dx.doi.org/10.4238/2015.October.28.14

ABSTRACT. Using mouse gene expression microarray analysis, we obtained dynamic expression profiles of the whole genome in a depilationinduced hair growth mouse model. S100A3 expression increased during the anagen phase and returned to normal during the telogen phase. The effects of S100A3 blockade on the hair growth cycle were examined in mice after subcutaneous injection of an anti-mouse S100A3 antibody. Protein localization of S100A3 was confined to the hair shafts during the anagen phase and the sebaceous glands during the telogen phase. S100A3 blockade delayed hair follicle entry into the anagen phase, decreased hair elongation, and reduced the number of hair follicles in the subcutis, which correlated with the downregulated expression of hair growth inductionrelated genes in vivo. The present study demonstrates that anti-S100A3 antibody inhibits mouse hair growth, suggesting that S100A3 can be used as a target for hair loss treatment.

Key words: S100A3; Anti-S100A3 antibody; Hair growth; Hair cycle; Anagen 


\section{INTRODUCTION}

Hair, a skin appendage, fulfills a wide range of requirements including physical protection, sensory functions, thermoregulation, and social interactions. A unique characteristic of hair growth is cyclicity, which involves a growth phase (anagen), an apoptosis-mediated regression phase (catagen), and a resting phase (telogen) (Müller-Röver et al., 2001; Schneider et al., 2009). Hair loss is a common and distressing problem. Although hair loss is not life-threatening, its profound impact on social interactions and on patients' psychological wellbeing is undeniable (Cotsarelis and Millar, 2001). Most currently marketed anti-hair loss products are ineffective, and only two (finasteride and minoxidil) have been approved by the U.S. Food and Drug Administration (D'Amico and Roehrborn, 2007). Therefore, novel pharmacological treatments and agents are in demand. Extensive studies have revealed diverse functional roles of several S100 proteins in a variety of cellular processes, such as cell cycle regulation, cell growth, cell differentiation, and mobility (Heizmann and Cox, 1998). Various human diseases are associated with deregulated expression of S100 genes, making them targets for future therapeutic interventions.

S100A3 (formerly named S100E) is a member of the S100 protein family, which is the largest subfamily of the EF hand type calcium-binding protein superfamily (Schäfer and Heizmann, 1996). It is unique among the $\mathrm{S} 100$ proteins in that it has the highest cysteine content (10\%) (Kizawa et al., 2013). It is specifically expressed in cuticular cells, and to a lesser extent in the cortical cells of hair follicles in humans (Kizawa et al., 1996; Takizawa et al., 1999), mice (Kizawa et al., 1998), and rats (Kizawa and Ito, 2005). In addition, S100A3 is expressed in head and neck cancer (Tyszkiewicz et al., 2014), breast cancer (Lloyd et al., 1996), gastric cancer (Liu et al., 2008), colorectal cancer (Liu et al., 2013), bladder cancer (Yao et al., 2007), and astrocytic tumors (Camby et al., 2000). Because these cell types are characterized by high proliferation rates, S100A3 is thought to be involved in cell cycle progression. The genomic organization of S100A3, S100A4, and S100A6 is conserved in mouse chromosome 3 (Kizawa et al., 1998). There are indications that S100A4 and S100A6 play important roles in the activation of stem cells at the onset of follicle regeneration and cell cycle withdrawal of the follicular stem cells (Ito and Kizawa, 2001). However, the role of S100A3 in the hair growth cycle is unknown.

Hair shafts, which are made by the hair follicle, undergo self-renewal when hairs are damaged. We proposed that homeostatically regulated gene expression during the hair cycle is essential for hair growth. Identification of the genes involved and characterization of their roles may lead to the discovery of the genome regulating hair growth. Therefore, we used a genome-wide gene expression array to screen for homeostatically regulated genes in a depilation-induced hair growth mouse model. The results show that S100A3 is an informative gene in hair regeneration. We cloned complementary DNA (CDNA) for mouse S100A3 and examined its expression profile. Injection of recombinant mouse S100A3 into the back skin of the mouse depilation model did not affect hair growth. We inferred that the S100A3 expression level in the depilated mouse was too high to be disturbed by exogenous S100A3. Therefore, we prepared an anti-S100A3 antibody and investigated its effect on mouse hair growth cycles. Our results indicate that S100A3 plays an important role in the anagen phase of the hair growth cycle.

\section{MATERIAL AND METHODS}

\section{Experimental animals}

All mouse experiments were conducted in accordance with the Guide for the Care 
and Use of Laboratory Animals (National Academies Press, Washington, DC, 1996), and were approved by the Animal Research Committee of Shanghai Jiao Tong University. Male C57BL/6 mice were purchased from Shanghai SLAC Laboratory Animal Co. (Shanghai, China). Mice were housed in a standard animal room with food and water ad libitum under controlled humidity and temperature $\left(22^{\circ} \pm 2^{\circ} \mathrm{C}\right)$ conditions. The room was illuminated by fluorescent lights from 8:00 am to $8: 00 \mathrm{pm}$.

\section{Protein purification of recombinant mouse S100A3}

We cloned the S100a3 gene from mouse skin, and inserted it into a pET28a (+) expression plasmid. The plasmid was transformed into Escherichia coli BL21 (DE3). The recombinant mouse S100A3 (rmS100A3) was overexpressed in bacteria upon induction by isopropyl $\beta$-Dthiogalactopyranoside. The recombinant protein was purified with Ni Sepharose (GE Healthcare, Pittsburgh, PA, USA). The His-Tag of the recombinant protein was cleaved with factor $\mathrm{Xa}$, and further purified by anion exchange using Q Sepharose Fast Flow (GE Healthcare) and sizeexclusion chromatography using Superdex 75 (GE Healthcare). The purified protein was sterilized by filtration through a low-binding $0.22-\mu \mathrm{m}$ filter.

\section{Production of anti-S100A3 antibody}

Specific antiserum for the S100A3 protein was obtained by immunizing rabbits with rmS100A3. Two mature male New Zealand white rabbits were immunized by four subcutaneous injections of rmS100A3 $(0.5 \mathrm{mg} / \mathrm{kg}$ body weight) with complete Freund's adjuvant. At intervals of 4 weeks, three booster immunizations were given with the emulsion prepared using incomplete Freund's adjuvant. The enzyme-linked immunosorbent assay titer of the collected antiserum rose to $6.4 \times 10^{5}$ against the rmS100A3 4 weeks after the first injection. Blood collection was performed 7 days following the third booster injection. The anti-S100A3 antibody and normal rabbit immunoglobulin $\mathrm{G}(\mathrm{lgG})$ were purified by Protein $\mathrm{G}$ affinity chromatography. Generally, a total of $12 \mathrm{mg}$ anti-S100A3 antibody was obtained from $5 \mathrm{~mL}$ antiserum. The purity of the antibody was determined to be higher than $90 \%$ by sodium dodecyl sulfate-polyacrylamide gel electrophoresis. The purified samples were sterilized by filtration through a $0.22-\mu \mathrm{m}$ filter. The purified anti-S100A3 antibody was used for the following immunohistochemical staining and blockade of S100A3 activity in vivo.

\section{Experimental study with anti-S100A3 antibody}

Twenty animals in two randomized groups $(N=10)$ were used for studying the activity of anti-S100A3 antibody. Anagen was induced by depilation of the back skin of 7-week-old C57BL/6 mice. This was done by applying a melted wax/rosin mixture to the back skin and peeling off this mixture after hardening, as previously described (Paus et al., 1990). All depilated mouse hair follicles were synchronized in the telogen phase. Animals in group 1 were subcutaneously injected with the anti-S100A3 antibody once a week for 3 consecutive weeks, and those in group 2 were injected with rabbit IgG as the control. The dose of the anti-S100A3 antibody was as previously described (Li et al., 2011). At 14 and 21 days, the mice were sacrificed to obtain skin specimens. Visible hair growth was recorded at 14 and 21 days. 


\section{Hair length determination}

Hairs were plucked from representative areas in the depilated dorsal center parts of mice at 14 and 21 days. We calculated the average hair length from 30 hairs per mouse.

\section{Histology and immunohistochemical staining}

Dorsal skins were harvested for histological analysis. The dorsal skins were fixed with $10 \%$ neutral formalin for routine histology, embedded in paraffin, and processed for hematoxylin and eosin (H\&E) staining. Dorsal skins were stained with rabbit anti-S100A3 antibody. The immunohistochemical analysis was performed using a diaminobenzidine Chromogen Kit (Boster, Wuhan, Hubei province, China), according to the manufacturer instructions.

\section{Hair follicle counting}

Digital photomicrographs were taken from representative areas of slides at a fixed magnification of $4 \mathrm{X}$. All images were cropped in a fixed area with a width of $1500 \mu \mathrm{m}$. We then manually counted hair follicles in the deep subcutis, as previously described (Zhang et al., 2013).

\section{Quantitative reverse transcription polymerase chain reaction (qRT-PCR)}

Total RNA was extracted from each snap-frozen skin sample using a two-step RNA extraction with TRIzol (Invitrogen, USA). Single-strand cDNA was synthesized using a PimeScript RT Reagent Kit (TaKaRa, China). Transcript expression was determined by qRT-PCR and normalized against $\beta$-actin. The primer sets used are shown in Table 1.

\begin{tabular}{|c|c|c|c|}
\hline Genes & Primer sequences & Product size (bp) & GenBank accession No. \\
\hline Foxe1 & $\begin{array}{l}\text { Forward: } 5^{\prime} \rightarrow 3^{\prime} \text { gacaaccccaagaagtggca } \\
\text { Reverse: } 5^{\prime} \rightarrow 3^{\prime} \text { ggatcttgaggaagcagtcg }\end{array}$ & 71 & NM_183298 \\
\hline Nog & $\begin{array}{l}\text { Forward: } 5^{\prime} \rightarrow 3^{\prime} \text { cctggtggacctcatcgaac } \\
\text { Reverse: } 5^{\prime} \rightarrow 3^{\prime} \text { gggggcgaagtagccataaa }\end{array}$ & 126 & NM_008711 \\
\hline Vcan & $\begin{array}{l}\text { Forward: } 5^{\prime} \rightarrow 3^{\prime} \text { tttacccgagttaccagactca } \\
\text { Reverse: } 5^{\prime} \rightarrow 3^{\prime} \text { ggagtagttgttacatccgttgc }\end{array}$ & 106 & NM_001081249 \\
\hline Ptch1 & $\begin{array}{l}\text { Forward: } 5^{\prime} \rightarrow 3^{\prime} \text { aaagaactgcggcaagttttg } \\
\text { Reverse: } 5^{\prime} \rightarrow 3^{\prime} \text { cttctcctatcttctgacgggt }\end{array}$ & 164 & NM_008957 \\
\hline
\end{tabular}

\section{Statistical analysis}

All data are reported as means \pm SD from three independent experiments. All statistical analyses were carried out using the SPSS 18.0 software. Differences between experimental groups were evaluated by Student's $t$-test. $P$ values less than 0.05 were considered to be statistically significant.

\section{RESULTS}

\section{Expression of S100A3 during the induced mouse hair cycle}

In our mouse model, a new cycle of hair growth was induced by depilation (Paus et al., 
1990). Briefly, a wax/rosin mixture was applied to the dorsal skin of 7-week-old C57BL/6 mice with all dorsal skin hair follicles in telogen, as evidenced by the homogeneous pink skin color. Removing the wax/rosin mixture removed all hair shafts and immediately induced homogeneous entry into the anagen phase; the consecutive phases of catagen and telogen were then entered spontaneously in a fairly homogeneous manner. Figure $1 \mathrm{~A}$ shows the morphology of the hair follicles at each time point after depilation. According to the classification of hair cycle phases in mice (Müller-Röver et al., 2001), 7 days mainly includes anagen III; 14 days, anagen VI; and 26 days, telogen. At 0 days, the hairs are in the telogen phase.

We performed DNA microarrays to profile mRNA expression in the adult mouse back skin from four representative time points after depilation. The expression of S100a3 increased during the anagen phase (0-16 days) and returned to normal during the telogen phase (26 days) (Figure 1B). The homeostatically regulated S100a3 gene in the expression array was confirmed by qRT-PCR (Figure 1C). The trend of S100A3 expression in the mouse depilated model was consistent with a previous report on the natural murine hair cycle (Lin et al., 2004). Taken together, the homeostatically regulated expression of S100A3 in the hair cycle suggests its participation in hair growth after depilation.

\section{A}

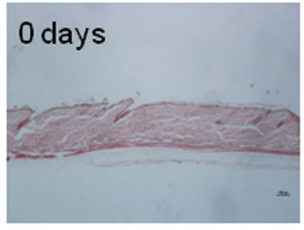

B

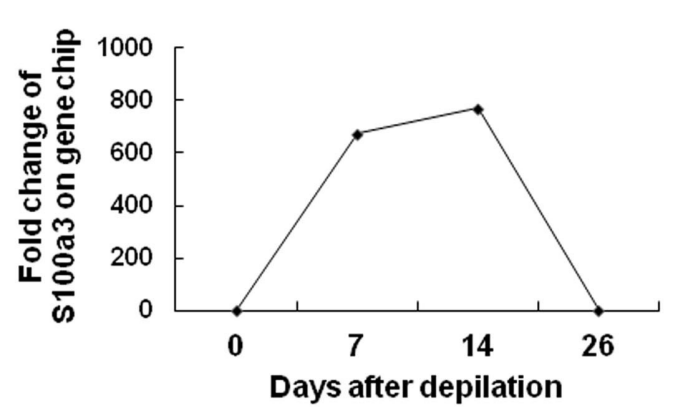

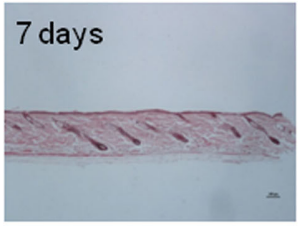
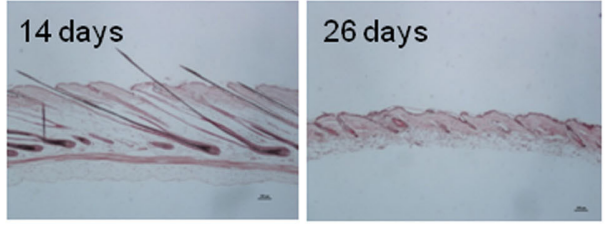

C

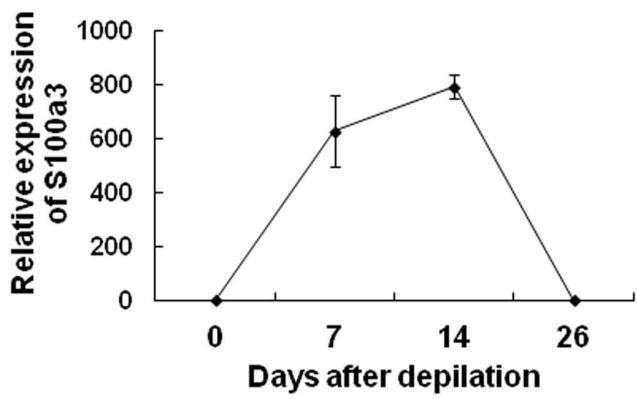

Figure 1. Expression profile of S100A3 at different phases of the hair growth cycle. Anagen was induced in the back skin of C57BL/6 mice by depilation. A. Sequential changes in hair follicle morphology after depilation. B. Signal intensity of S100a3 mRNA by gene expression array. C. Expression of S100a3 by quantitative reverse transcription polymerase chain reaction (qRT-PCR) relative to their baseline at 0 days. " 0 days" indicates telogen hair before depilation. Scale bar: $100 \mu \mathrm{m}$.

\section{Localization of S100A3 protein during the induced mouse hair cycle}

Immunohistochemical staining was used to confirm S100A3 protein expression. Rabbit anti-mouse S100A3 polyclonal antibody (anti-S100A3) was produced and purified as described in the Material and Methods section. 
As shown in Figure 2B, C, F, and G, S100A3 was highly expressed in the cuticle of the hair shafts at 7 and 14 days (the anagen phase of the hair cycle). This observation is consistent with a previous report focused solely on S100A3 expression in developing hair follicles (Kizawa et al., 1998). In addition, we initially found that S100A3 protein was present in the sebaceous glands during the telogen phase (Figure 2A, D, E, and H). Therefore, S100A3 protein is abundant in the local hair follicle region only during the anagen phase of the hair cycle. The results indicate that our antibody is capable of specifically recognizing its target S100A3 protein in mouse skin tissues, and that S100A3 may play an important role at the onset of follicle regeneration.
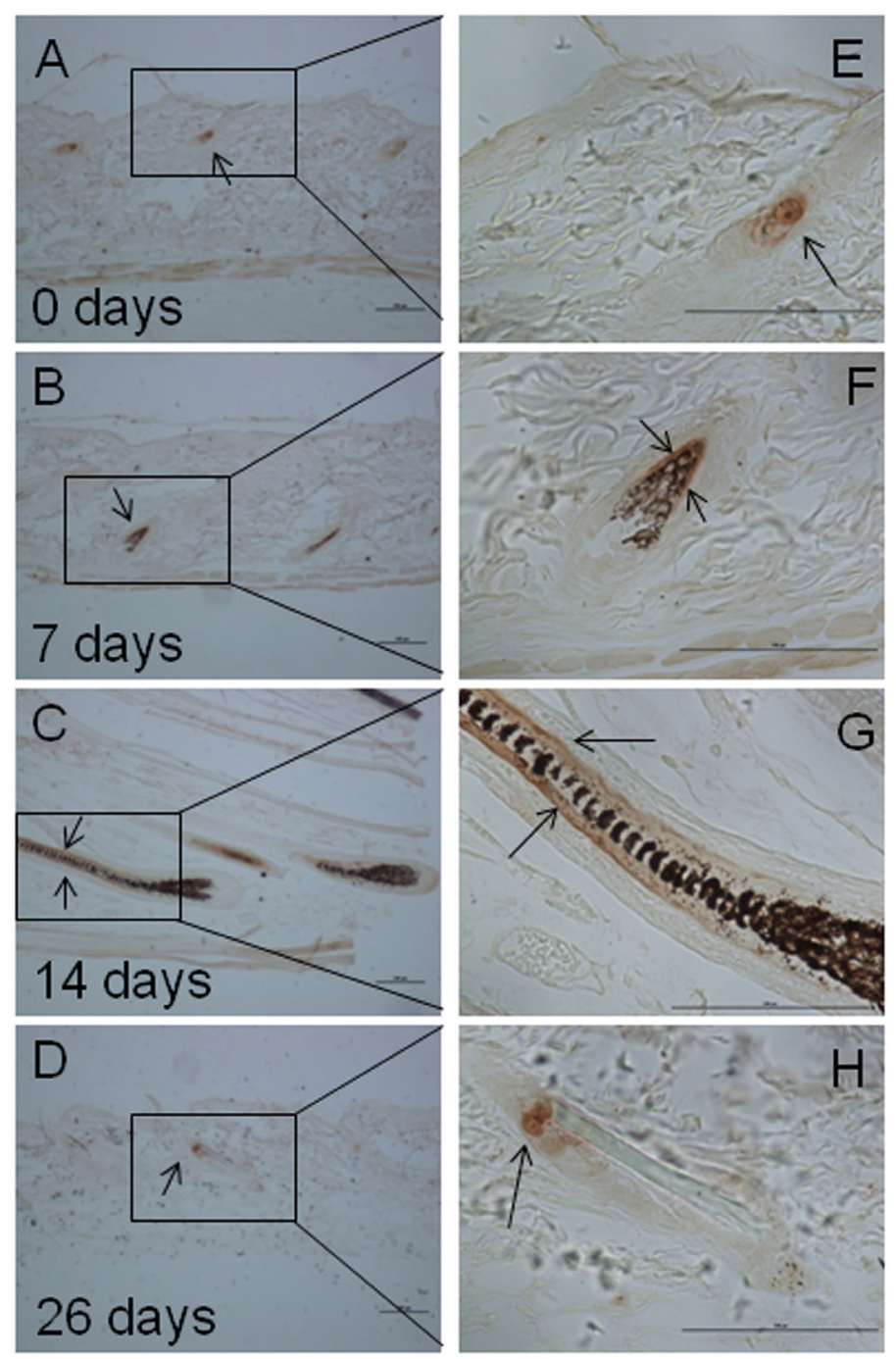

Figure 2. Localization of S100A3 at different phases of the hair growth cycle. Immunohistochemical analysis was used to monitor the S100A3 protein (arrows) in the longitudinal sections of dorsal skin. A.-D. S100A3 was observed in the sebaceous glands and hair shafts at 0, 7, 14, and 26 days after depilation. E.-H. Magnified photographs of the framed labeled area. Scale bar: $100 \mu \mathrm{m}$. 


\section{Effect of anti-S100A3 antibody on hair growth}

To examine whether S100A3 expression is essential to hair growth, the anti-S100A3 antibody was used to block S100A3 function when the anagen phase of the hair cycle was induced. Adult C57BL/6 mice are useful for screening hair growth-promoting agents because their truncal pigmentation is dependent on their follicle melanocytes, which produce pigment only during anagen (Plonka et al., 2005). The 7-week-old C57BL/6 mice were subcutaneously administered with the anti-S100A3 antibody or with normal rabbit IgG (controls), and 1 day after the first antibody injection, the hair cycle was induced by depilation. The mice were treated with the anti-S100A3 antibody or normal rabbit IgG once a week, for 3 weeks. It has been reported that the skin color of the mice in the telogen phase is pink and gradually becomes gray and black along with anagen initiation (Datta et al., 2009). At 7 days after depilation, the skin of the anti-S100A3 antibodytreated mice was still pink, while the skin of the rabbit IgG-treated mice had turned gray (Figure 3). The results indicate that the application of anti-S100A3 antibody delayed commencement of the anagen phase of the hair follicles in the mice after depilation.

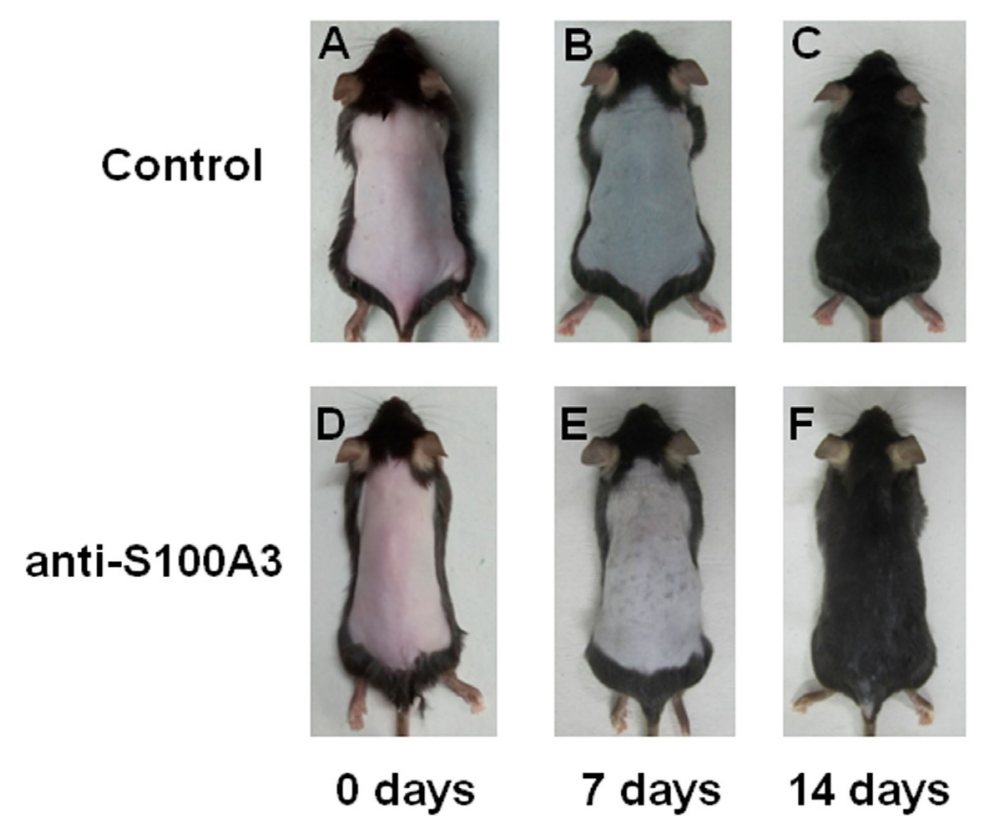

Figure 3. Inhibition of anagen induction from telogen by anti-S100A3 antibody in C57BL/6 mice. Telogen-matched, 7-week-old C57BL/6 mice were depilated and subcutaneously injected with rabbit lgG as controls $(\mathrm{N}=10)$ or antiS100A3 antibody $(N=10)$ once weekly for 3 weeks. Photographs were taken at 0,7 , and 14 days. Darker coloration was observed in the control mice compared with the anti-S100A3 antibody-treated mice at 7 days after depilation. Control mice (A.-C.); anti-S100A3 antibody-treated mice (D.-F.).

\section{Effects of anti-S100A3 antibody on hair follicle number and hair shaft elongation}

An increase in the number of hair follicles is considered an indicator of the transition from the telogen to the anagen phase. The anagen phase is associated with an increase in the number of hair follicles in the deep subcutis compared with the telogen phase, where hair follicles are 
present in the dermis only (Datta et al., 2009; Zhang et al., 2013). The effect of anti-S100A3 on the density of hair follicles was further assessed by the histological analysis of the mouse skin samples after staining with H\&E. In the longitudinal and transverse sections of the skin, we observed that the number of hair follicles in the deep dermal areas of the anti-S100A3 antibody-treated group was significantly lower than in the control group (Figure 4).

\section{Control}
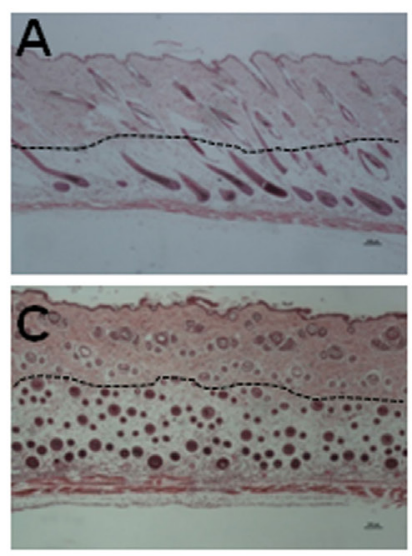

E
anti-S100A3
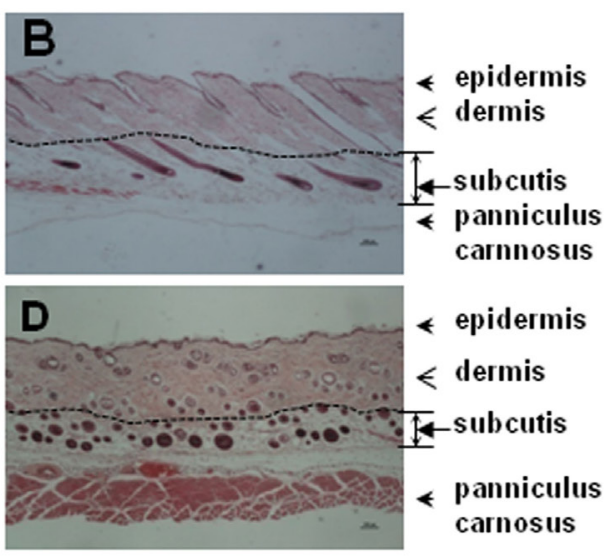

« epidermis

$<$ dermis

L-subcutis

$\leftarrow \begin{aligned} & \text { panniculus } \\ & \text { carnosus }\end{aligned}$

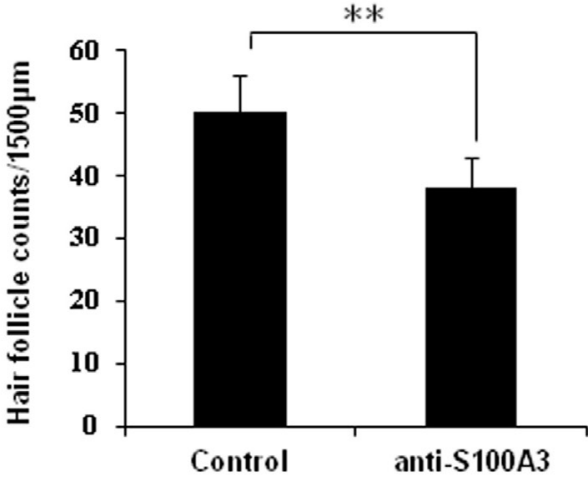

14 days

Figure 4. Anti-S100A3 antibody decreased the number of hair follicles in C57BL/6 mice. The effect of anti-S100A3 antibody on the hair follicles was analyzed using hematoxylin and eosin staining at 14 days after depilation. A. B. Longitudinal sections of the dorsal skins. C. D. Transverse sections of the dorsal skins. Scale bar: $100 \mu \mathrm{m}$. E. Number of hair follicles in deep subcutis. Values are reported as means $\pm \mathrm{SD}\left(\mathrm{N}=30 /\right.$ mouse; ${ }^{* *} \mathrm{P}<0.01$, vs control).

To confirm whether anti-S100A3 antibody inhibited hair growth, photographs were taken at 14 days after subcutaneously injecting anti-S100A3 antibody or rabbit IgG. In addition, we measured the length of 30 hairs plucked from the dorsal skin of each mouse at 14 and 21 days. As shown in Figure 5 , the hairs in the anti-S100A3 antibody-treated group were significantly shorter than those in the control group $(P<0.01)$. Taken together, subcutaneous injection of anti-S100A3 antibody significantly decreased the number of hair follicles entering anagen, and inhibited the elongation of hair shafts. 

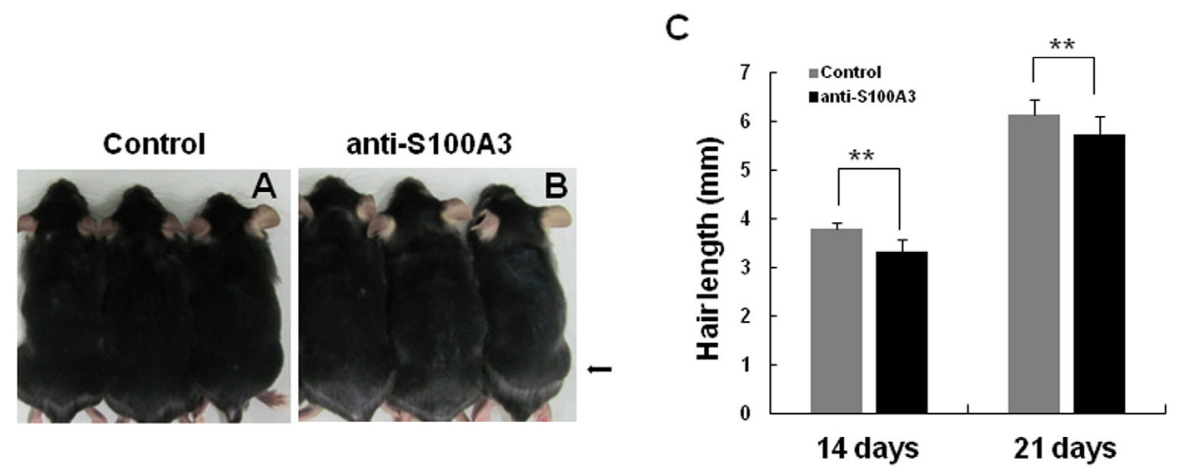

Figure 5. Hair length at 14 and 21 days after depilation. Telogen-matched, 7-week-old C57BL/6 mice were depilated and subcutaneously injected with anti-S100A3 antibody $(\mathrm{N}=5)$, or rabbit lgG as control $(\mathrm{N}=5)$. A. B. Back skins were photographed at 14 days after depilation. The image shown is a representative picture of five mice. C. Hair length at different time intervals after antibody treatment. Data are reported as means $\pm S D\left({ }^{*} P<0.01\right.$, vs control).

\section{Downregulation of the hair growth-related genes with anti-S100A3 antibody}

Previous studies have shown that activation of the Wnt/ $\beta$-catenin, Shh, and bone morphogenetic protein signaling pathways is critical for anagen induction and hair growth (Cotsarelis and Millar, 2001; Schneider et al., 2009; Jiang et al., 2010). Foxe1 and Ptch1 are downstream target genes of the Shh pathway in hair growth (Brancaccio et al., 2004). Noggin (Nog) and Versican (Vcan) are indicated as hair induction-related genes (Botchkarev et al., 1999; Dong et al., 2014). We therefore isolated total RNA from C57BL/6 skin samples at 14 days after depilation and examined expression levels of hair growth-related genes (Foxe1, Nog, Vcan, and Ptch1) by qRT-PCR. We observed that the expression levels of Foxe1, Nog, Vcan, and Ptch1 were lower in the anti-S100A3 antibody-treated mice than in the controls (Figure 6).

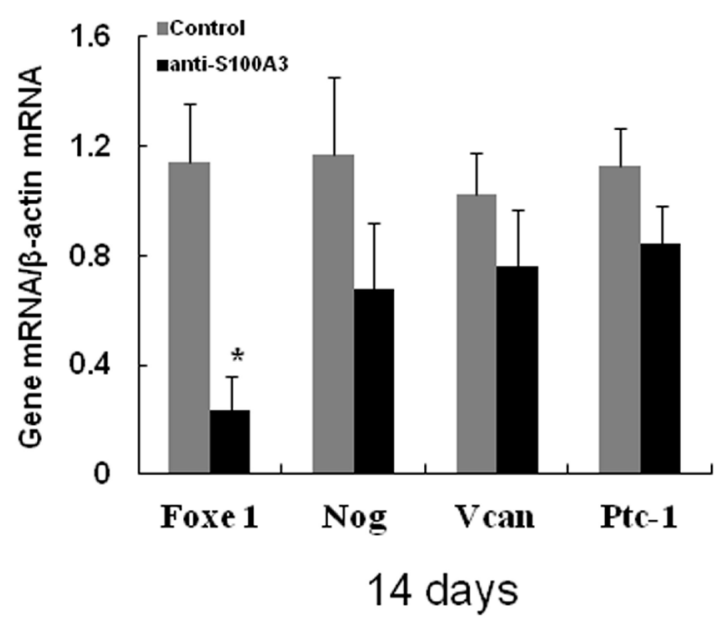

Figure 6. Hair growth-related gene expression in the depilated skin of mice at 14 days. Relative mRNA expression levels of Foxe1, Nog, Vcan, and Ptch1 were measured by quantitative reverse transcription polymerase chain reaction (qRT-PCR) with specific primers ( ${ }^{*} P<0.05, v s$ control, $N=4$ ). Data are reported as means $\pm S D$ for three independent runs performed on the C57BL/6 mice. 
It has been reported that Foxe1 is crucial for correct hair follicle orientation in the dermis and subcutis (Brancaccio et al., 2004). Our expression data suggest that S100A3 is important for the expression of hair growth-related genes.

\section{DISCUSSION}

This study focused on the localization of S100A3 protein and the effects of anti-S100A3 antibody on hair regeneration. As with a previous report (Kizawa et al., 1996), we observed strong expression of S100A3 in the hair shafts during the anagen phase of the hair growth cycle in the mouse depilation model. In addition, we initially found that S100A3 was expressed in the sebaceous glands during telogen. Furthermore, the blockade of S100A3 activity with anti-S100A3 antibody delayed hair growth after depilation, and reduced the number of hair follicles in the subcutis, which corresponded to the downregulation of hair growth-related genes in the mouse model of hair regeneration.

The S100 proteins comprise a family of 24 low molecular weight (9-13 kDa) proteins, 13 of which (S100A2, S100A3, S100A4, S100A6, S100A7, S100A8, S100A9, S100A10, S100A11, S100A12, S100A15, S100B, and S100P) are expressed in the epidermis (Eckert et al., 2004; Donato et al., 2013). S100A4 is expressed in the bulge region of the epithelial portion. S100A6 is continuously distributed from the innermost cell layer of the outer root sheath to the bulge area. The genomic organization of S100A3, S100A4, and S100A6 is conserved in mouse chromosome 3. The expression of S100A3 is complementary to that of S100A6 in the matrix daughter cells of the anagen hair follicle. S100A6 takes part in the activation of follicular stem cells (Kizawa et al., 1998; Ito and Kizawa, 2001). However, whether S100A3 participates in hair follicle regeneration is unknown. The mouse S100a3 gene was specifically expressed in dorsal skin containing hair follicles. Previous studies suggest a close association between S100a3 and epithelial differentiation leading to hair shaft formation (Takizawa et al., 1999; Ito and Kizawa, 2001). In this study, we found that the S100A3 protein appeared in the sebaceous glands at telogen and the cuticles of the hair shafts at anagen (Figure 2). The expression of S100A3 was induced by depilation. The level of S100A3 mRNA was elevated during the transition from telogen to anagen in the hair cycle, and returned to the baseline level following the subsequent telogen (Figure 1). Therefore, the S100A3 expression pattern during the hair cycle suggests its role in hair regrowth induced by depilation.

Calcium is an important divalent cation that operates within the cellular system. S100 proteins may play a role in the regulation of the intracellular calcium level, which is often connected to critical cellular events, such as proliferation, differentiation, or apoptosis (Kizawa et al., 1996). In addition, several S100 proteins act as intracellular regulators and as extracellular signaling proteins. Within cells, S100 proteins are involved in the regulation of proliferation, differentiation, apoptosis, inflammation, and migration/invasion. As extracellular signaling molecules, S100 proteins exert regulatory activities on macrophages, lymphocytes, mast cells, and epithelial cells. Therefore, they participate in the immune response, cell migration and chemotaxis, tissue repair, and tumor cell invasion (Donato et al., 2013). S100A3 is overexpressed in head and neck cancer, colorectal cancer, bladder cancer, gastric cancer, and astrocytic tumors, but has an inverse correlation to breast cancer progression (Lloyd et al., 1996; Camby et al., 2000; Yao et al., 2007; Liu et al., 2008, 2013; Tyszkiewicz et al., 2014). It is also highly expressed in hair root cells, and is thought to have a role in epithelial cell differentiation and $\mathrm{Ca}^{2+}$-dependent hair cuticular barrier formation (Kizawa et al., 2008). The present study demonstrated that subcutaneous injection with anti-S100A3 antibody led to hair growth retardation and a reduced number of hair follicles (Figures 3-5). Importantly, the 
loss of S100A3 during daily life results in the delamination of cuticles, especially in distal hair (Inoue et al., 2000). These findings suggest that S100A3 participates in the regulation of the hair cycle, and is associated with hair growth and formation.

It is known that various growth factor, cytokines, hormones, adhesion molecules, and related enzymes are involved in hair growth (Stenn and Paus, 2001). Vascular endothelial growth factor promotes hair follicle growth through induced angiogenesis (Yano et al., 2001). Insulinlike growth factor- 1 is expressed in the hair follicle and affects follicle proliferation and the hair growth cycle (Hayashi et al., 2013). Hepatocyte growth factor (HGF) drives telogen follicles into anagen, and prolongs its duration. Local injection of HGF promotes hair follicle growth and delays the transition from anagen to telogen (Jindo et al., 1998; Paus and Foitzik, 2004). Transforming growth factor $\beta 1$ (TGF- $\beta 1$ ) is an inhibitor of keratinocyte proliferation and an inducer of keratinocyte apoptosis. Injection of TGF- $\beta 1$ into the back skin of mice induces premature entry into the catagen phase (Foitzik et al., 2000). S100A4 and S100A6 proteins are present in the bulge region during hair cycle transition. S100A6 participates in cell cycle withdrawal of follicular stem cells (Ito and Kizawa, 2001). In this study, we found that S100A3 was highly expressed in anagen, especially in anagen VI (14 days). Anti-S100A3 antibody inhibited hair follicle entry into the anagen phase and decreased hair elongation. In addition, it decreased the expression of hair growth-related genes; in particular, the mRNA level of Foxe1 decreased by 4.9-fold. A previous study established Foxe1 as a downstream target of the Shh/Gli pathway in hair follicle morphogenesis, and as a crucial component in the correct orientation of hair follicles in the dermis and subcutis (Brancaccio et al., 2004), which supports our observation of the reduced number of hair follicles in the subcutis. Our study shows that S100A3 treatment does not affect hair growth in the mouse depilation model (data not shown). It might be that the S100A3 expression level is too high in vivo to be disturbed at anagen by exogenous S100A3.

In summary, we have shown that S100A3 is present in hair shafts and sebaceous glands. Neutralization of S100A3 delays the initiation of the hair follicle transition from the telogen to the anagen phase, inhibits hair growth rates, decreases the number of hair follicles, and downregulates expression of hair growth-related genes in vivo. Our findings indicate that S100A3 plays an important role in hair regeneration, but further studies are required to fully reveal the molecular basis of its function in hair growth.

\section{Conflicts of interest}

The authors declare no conflict of interest.

\section{ACKNOWLEDGMENTS}

Research supported by the National Natural Science Foundation of China (\#81273573) and the Science and Technology Commission of Shanghai Municipality (\#11431921300).

\section{REFERENCES}

Botchkarev VA, Botchkareva NV, Roth W, Nakamura M, et al. (1999). Noggin is a mesenchymally derived stimulator of hairfollicle induction. Nat. Cell Biol. 1: 158-164.

Brancaccio A, Minichiello A, Grachtchouk M, Antonini D, et al. (2004). Requirement of the forkhead gene Foxe1, a target of sonic hedgehog signaling, in hair follicle morphogenesis. Hum. Mol. Genet. 13: 2595-2606. 
Camby I, Lefranc F, Titeca G, Neuci S, et al. (2000). Differential expression of S100 calcium-binding proteins characterizes distinct clinical entities in both WHO grade II and III astrocytic tumours. Neuropathol. Appl. Neurobiol. 26: 76-90.

Cotsarelis G and Millar SE (2001). Towards a molecular understanding of hair loss and its treatment. Trends Mol. Med. 7: 293-301.

D'Amico AV and Roehrborn CG (2007). Effect of $1 \mathrm{mg} /$ day finasteride on concentrations of serum prostate-specific antigen in men with androgenic alopecia: a randomised controlled trial. Lancet Oncol. 8: 21-25.

Datta K, Singh AT, Mukherjee A, Bhat B, et al. (2009). Eclipta alba extract with potential for hair growth promoting activity. J. Ethnopharmacol. 124: 450-456.

Donato R, Cannon BR, Sorci G, Riuzzi F, et al. (2013). Functions of S100 proteins. Curr. Mol. Med. 13: 24-57.

Dong L, Hao H, Xia L, Liu J, et al. (2014). Treatment of MSCs with Wnt1a-conditioned medium activates DP cells and promotes hair follicle regrowth. Sci. Rep. 4: 5432.

Eckert RL, Broome AM, Ruse M, Robinson N, et al. (2004). S100 proteins in the epidermis. J. Invest. Dermatol. 123: 23-33.

Foitzik K, Lindner G, Mueller-Roever S, Maurer M, et al. (2000). Control of murine hair follicle regression (catagen) by TGFbeta1 in vivo. FASEB J. 14: 752-760.

Hayashi Y, Yamamoto N, Nakagawa T and Ito J (2013). Insulin-like growth factor 1 inhibits hair cell apoptosis and promotes the cell cycle of supporting cells by activating different downstream cascades after pharmacological hair cell injury in neonatal mice. Mol. Cell Neurosci. 56: 29-38.

Heizmann CW and Cox JA (1998). New perspectives on S100 proteins: a multi-functional Ca(2+)-, Zn(2+)- and Cu(2+)-binding protein family. Biometals 11: 383-397.

Inoue T, Sasaki I, Yamaguchi M and Kizawa K (2000). Elution of S100A3 from hair fiber: New model for hair damage emphasizing the loss of S100A3 from cuticle. J. Cosmet. Sci. 51: 15-25.

Ito $\mathrm{M}$ and Kizawa K (2001). Expression of calcium-binding S100 proteins A4 and A6 in regions of the epithelial sac associated with the onset of hair follicle regeneration. J. Invest. Dermatol. 116: 956-963.

Jiang S, Zhao L, Teklemariam T and Hantash BM (2010). Small cutaneous wounds induce telogen to anagen transition of murine hair follicle stem cells. J. Dermatol. Sci. 60: 143-150.

Jindo T, Tsuboi R, Takamori K and Ogawa H (1998). Local injection of hepatocyte growth factor/scatter factor (HGF/SF) alters cyclic growth of murine hair follicles. J. Invest. Dermatol. 110: 338-342.

Kizawa K and Ito M (2005). Characterization of epithelial cells in the hair follicle with S100 proteins. Methods Mol. Biol. 289: 209-222.

Kizawa K, Uchiwa H and Murakami U (1996). Highly-expressed S100A3, a calcium-binding protein, in human hair cuticle. Biochim. Biophys. Acta 1312: 94-98.

Kizawa K, Tsuchimoto S, Hashimoto K and Uchiwa H (1998). Gene expression of mouse S100A3, a cysteine-rich calciumbinding protein, in developing hair follicle. J. Invest. Dermatol. 111: 879-886.

Kizawa K, Takahara H, Troxler H, Kleinert P, et al. (2008). Specific citrullination causes assembly of a globular S100A3 homotetramer: a putative $\mathrm{Ca}^{2+}$ modulator matures human hair cuticle. J. Biol. Chem. 283: 5004-5013.

Kizawa K, Jinbo Y, Inoue T, Takahara H, et al. (2013). Human S100A3 tetramerization propagates $\mathrm{Ca}(2+) / \mathrm{Zn}(2+)$ binding states. Biochim. Biophys. Acta 1833: 1712-1719.

Li J, Gao J, Yan D, Yuan Y, et al. (2011). Neutralization of chemokine CXCL14 (BRAK) expression reduces CCI4 induced liver injury and steatosis in mice. Eur. J. Pharmacol. 671: 120-127.

Lin KK, Chudova D, Hatfield GW, Smyth P, et al. (2004). Identification of hair cycle-associated genes from time-course gene expression profile data by using replicate variance. Proc. Natl. Acad. Sci. U. S. A. 101: 15955-15960.

Liu J, Li X, Dong GL, Zhang HW, et al. (2008). In silico analysis and verification of S100 gene expression in gastric cancer. BMC Cancer 8: 261.

Liu B, Sun WY, Zhi CY, Lu TC, et al. (2013). Role of S100A3 in human colorectal cancer and the anticancer effect of cantharidinate. Exp. Ther. Med. 6: 1499-1503.

Lloyd BH, Ruddell C, Rudland PS and Barraclough R (1996). S100A3 mRNA expression displays an inverse correlation to breast cancer progression. Biochem. Soc. Trans. 24: 340S.

Müller-Röver S, Handjiski B, van der Veen C, Eichmüller S, et al. (2001). A comprehensive guide for the accurate classification of murine hair follicles in distinct hair cycle stages. J. Invest. Dermatol. 117: 3-15.

Paus R and Foitzik K (2004). In search of the "hair cycle clock": a guided tour. Differentiation 72: 489-511.

Paus R, Stenn KS and Link RE (1990). Telogen skin contains an inhibitor of hair growth. Br. J. Dermatol. 122: 777-784.

Plonka PM, Michalczyk D, Popik M, Handjiski B, et al. (2005). Splenic eumelanin differs from hair eumelanin in C57BL/6 mice. Acta Biochim. Pol. 52: 433-441.

Schäfer BW and Heizmann CW (1996). The S100 family of EF-hand calcium-binding proteins: functions and pathology. Trends Biochem. Sci. 21: 134-140. 
Schneider MR, Schmidt-Ullrich R and Paus R (2009). The hair follicle as a dynamic miniorgan. Curr. Biol. 19: R132-R142. Stenn KS and Paus R (2001). Controls of hair follicle cycling. Physiol. Rev. 81: 449-494.

Takizawa T, Takizawa T, Arai S, Kizawa K, et al. (1999). Ultrastructural localization of S100A3, a cysteine-rich, calcium binding protein, in human scalp hair shafts revealed by rapid-freezing immunocytochemistry. J. Histochem. Cytochem. 47: 525-532.

Tyszkiewicz T, Jarzab M, Szymczyk C, Kowal M, et al. (2014). Epidermal differentiation complex (locus 1q21) gene expression in head and neck cancer and normal mucosa. Folia Histochem. Cytobiol. 52: 79-89.

Yano K, Brown LF and Detmar M (2001). Control of hair growth and follicle size by VEGF-mediated angiogenesis. J. Clin. Invest. 107: 409-417.

Yao R, Lopez-Beltran A, Maclennan GT, Montironi R, et al. (2007). Expression of S100 protein family members in the pathogenesis of bladder tumors. Anticancer Res. 27: 3051-3058.

Zhang NN, Park DK and Park HJ (2013). Hair growth-promoting activity of hot water extract of Thuja orientalis. BMC Complement. Altern. Med. 13: 9. 